Review Articles

\title{
Mitochondria are Naturally Micro Robots - A review
}

\author{
${ }^{1}$ Raffaella Aversa, ${ }^{2}$ Relly Victoria V. Petrescu, ${ }^{1}$ Antonio Apicella and ${ }^{2}$ Florian Ion T. Petrescu \\ ${ }^{I}$ Advanced Material Lab, Department of Architecture and Industrial Design, \\ Second University of Naples, 81031 Aversa (CE) Italy \\ ${ }^{2}$ IFToMM, ARoTMM, Bucharest Polytechnic University, Bucharest, (CE) Romania
}

Article history

Received: 10-11-2016

Revised: 11-11-2016

Accepted: 15-11-2016

Corresponding Author: Florian Ion T. Petrescu IFToMM, ARoTMM,

Bucharest Polytechnic

University, Bucharest, (CE)

Romania

Email: petrescuflorian@yahoo.com

\begin{abstract}
The mitochondrion is a natural robot (cell) with a length of about one micron. Between organisms (plant, animal or human) with its organs and the atomic and molecular components are positioned based cells, which are building all the necessary organs body. Years ago, medical specialists who are studying organ disease have considered that is caused by impaired organ or related to other organs. For example, it was considered that all nerve diseases have started from stress due to disorder of the hypothalamic-pituitary-adrenal (adrenal glands). Today one knows that psychological stress triggers some metabolic, inflammatory and transcriptional perturbations that ultimately dispose to malady in cellular energetics, involving mitochondrial energy production respiratory stress. Future research on mitochondrial can lead to the elucidation of great issues of life (including the generation of illness, aging and death bodies). The main idea of this paper is that mitochondria are independent cells, flexible, essential for complex life.
\end{abstract}

Keywords: Biomaterials, Bioengineering, Biotechnologies, Mitochondrion, ATP Molecule, Biochemical Processes, Mitochondrial Energy Production, Transcriptional Perturbations, Predispose to Diseases, Oxidative Stress, Tissue Engineering

\section{Introduction}

Mitochondrion is a cell-organ found in massive numbers in most cells, in which the biochemical processes of respiration and production of energy occur. It has a membrane double, the inner layer being folded inward to form layers (cristae). Chloroplasts are cellorgans similar to the mitochondria of animal cells. Mitochondrion has a length of about one micron. Mitochondrion is a cell in cell! A mitochondrion has got all needed for an independent cell: Two membranes for protection, mitochondrial intermembrane space, crests, ribosomes (consisting of a minute particle RNA and associated proteins, which can be found in massive numbers in the living cells cytoplasm. THEY bind messenger RNA and transfer RNA to synthesize Polypeptides and proteins. Motors, RNA polymerases and ribosomes Such as, produce trailing strands of mRNA and protein, respectively.

A mitochondrial cell may live free, even though it prefers to live in symbiosis within other cells. Other eukaryotic cells from animal and human need more mitochondrial cells to live. Without these microcells the animal and human (eukaryotic) cells can't live! Another important function of a mitochondrion is to kill eukaryotic cells when it is their time to die. If a eukaryotic cell can multiply by 40 times when multiplied by 39 times the mitochondria present in it already begin to prepare the eukaryotic cell for death. They begin to leave the eukaryotic cell to perdition, not to die with her and to no longer supply her with power. If one eukaryotic cell is sick the inside mitochondrial cells are trying to heal it first, but if this is not possible then the eukaryotic cell is marked and all mitochondria inside begin the process to kill her even the eukaryotic cell still has many multiplication cycles possible. If eukaryotic cell (dying, abandoned by mitochondria) do not dies quickly, turns into a diseased cell cancer (harmful to the body). The same undesirable phenomenon can happen when they get sick mitochondrial cells itself.

Today, we know many causes that can cause mitochondrial disorders (Andrews and Walker, 1999; Arkblad et al. 1996; Bandura et al., 1985; Booth et al., 2008; Bornstein et al., 2008; Bullitt, 1990; Buzea et al., 2015; Cohen et al., 2007; DeSantis et al., 2015; Dimsdale and Moss, 1980; Dimsdale and Herd, 1982; 
Gimsa et al., 2009; Gómez-Durán et al., 2010; Gonzalez-Bono et al., 2002; Gorman et al., 2015; Gray et al., 2014; Gut and Verdin, 2013; Hunter et al., 2012; Jeppesen et al., 2013; Jones and Gilligan, 1983; Juster et al., 2012; Kado et al., 1999; Koopman et al., 2012; Lambert and Brand, 2009; Livak and Schmittgen, 2001; Lundberg, 2005; Wallace et al., 2010; Rangaraju et al., 2014; Manoli et al., 2007; McEwen, 1998a; 1998b; 2007; McFarland et al., 2010; Meimaridou et al., 2013; Morava and Kozicz, 2013; Nasca et al., 2015; Olsen et al., 2013; Pelletier et al., 2012; Petrescu et al., 2015; Petrescu and Petrescu, 2015; Picard and Turnbull, 2013; Picard et al., 2014a; Picard and McEwen, 2014; Picard et al., 2014b; 2015; Rohleder, 2014; Saudubray and Rabier, 2007; Schroeder et al., 2013; Sindi et al., 2013; Singh et al., 1999; Sun et al., 2013; Szendroedi et al., 2012; Ulrich-Lai and Herman, 2009; Vos et al., 2010; Weiner, 1992; Wellen et al., 2009; Wallace, 2013; Xia et al., 2012; Ye et al., 2014; Zhang et al., 2010; Zhou et al., 2007). Between organism (plant, animal or human) with its organs and the atomic and molecular components are positioned based cells, which are building all the necessary organs body. Any base cell has his live and tries to feed herself and multiply. A base cell can't see the mother organ or the organism. Moreover sometimes stubborn attempt to survive of a basic cell can cause disease to organ and later reach the whole body. Years ago the medical specialists studying organ disease have considered that is caused by the impaired organ or related to other organs. For example, it was considered that all nerve diseases have starting from stress due to disorder of the hypothalamic-pituitary-adrenal (adrenal glands). Today one knows that psychological stress triggers some metabolic, inflammatory and transcriptional perturbations that ultimately dispose to malady in cellular energetics, involving mitochondrial energy production and respiratory stress.

All future research on mitochondrial, may lead tomorrow to elucidation of great issues of life (including the generation of illness, aging and death bodies).

The main idea of this paper is that mitochondria are independent cells, flexible, essential for complex life. Today we not yet know the exact origin of these cells miraculous.

It looks like a mitochondrion is a bacterium independent complex that has adapted easily to life in symbiosis with eukaryotic cells.

As every mitochondrion of an organism has its own genetic code, it is not practically possible transfer of mitochondria from one individual to another. Therefore all ads that promise this are just a delusion. The study of mitochondria helps us to achieve spectacular results in preventing aging in humans, much more than conventional methods used so far (Buzea et al., 2015; Petrescu et al., 2015).

\section{First Energy Source of Life}

An obscure compound which is known as pyrophosphite could have been a source of energy that allowed the first life on Earth to form (New Theory for Life's First Energy Source, Corey Zah) (Petrescu and Petrescu, 2015).

The author suggests that pyrophosphate would have been relevant in the shift from basic chemistry to complex biology when life on earth began.

They even brought further evidence of the importance of this molecule and plan to further investigate its role in abiogenesis-that is how life on Earth emerged from inanimate matter billions of years ago. In reality there are several conflicting theories of abiogenesis, each trying to bring something new about how life arose on Earth.

What is essential in these studies eventually is the energy. Living matter has constantly need energy to exist and function.

The main source of energy of living matter is made in molecules known as ATP (Adenosine triphosphate).

An ATP molecule may change any sunlight heat to a form of energy which may be used by plants, humans and animals.

An ATP molecule contains these four vital elements: Oxygen, hydrogen, nitrogen and phosphorus (thirteen oxygen atoms, eight hydrogen atoms, five nitrogen atoms and three phosphorous atoms).

Practically is important how the atoms of the four elements are connected in an ATP molecule (Fig. 1).

ATP is constantly used and regenerated in cells via a process known as respiration, a process driven by natural catalysts called enzymes.

ATP transports chemical energy within cells in order to achieve metabolic processes.

It is one of the final cell respiration and fermentation and is used by enzymes and structural proteins in many cellular processes, such as: Motility, biosynthetic reactions and cell division.

One molecule of ATP contains three phosphate groups, which is produced by a great variety of enzymes, including the synthesis of ATP to Adenosine Diphosphate (ADP), Adenosine Monophosphate (AMP) and different donors of phosphate group.

Metabolic processes that use ATP as Associate in energy supply, convert it back then to its precursors. In this mode ATP is continually recycled in organisms.

The human body contains for example at one time an amount of about $250 \mathrm{~g}$ of ATP (the equivalent of a single AA battery).

ATP is employed as a substrate in signal transduction pathways by kinases that phosphorylate proteins and lipids. It's additionally employed by adenylate cyclase that uses ATP to provide the second traveller molecule cyclic AMP. The magnitude relation between ATP and 
AMP is employed as how for a cell to sense what proportion energy is out there and management the metabolic pathways that manufacture and consume ATP.<smiles>Nc1ncnc2c1ncn2C1OCC(O)C1OP(=O)(O)OP(=O)(O)OP(=O)(O)O</smiles>

Fig. 1. How the atoms of the four elements are connected on one ATP molecule

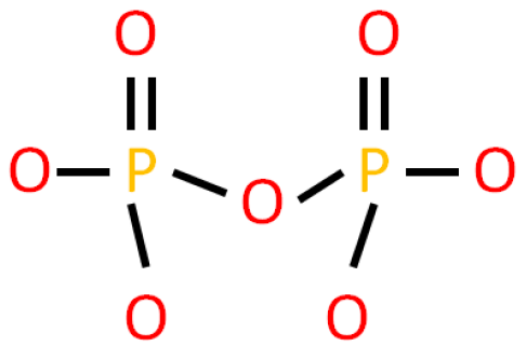

Fig. 2. A pyrophosphate anion having structure "P2O74-"

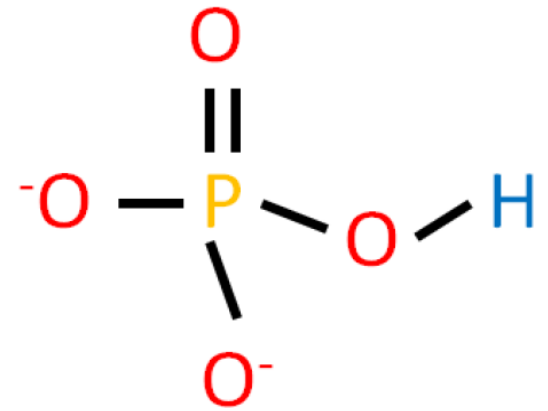

Fig. 3. Hydrogen phosphate, HPO42- (notted with Pi)

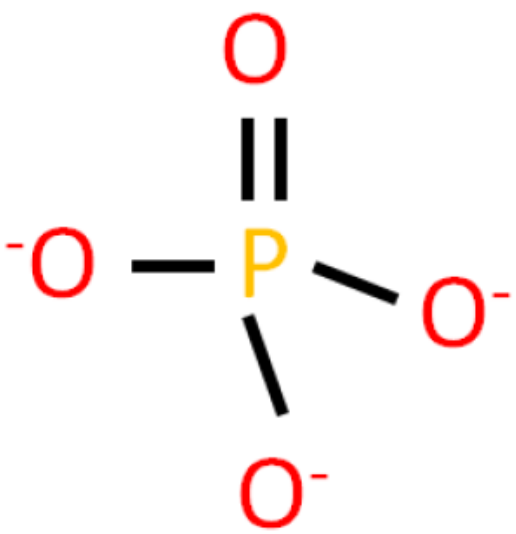

Fig. 4. One phosphate ion
With the exception of its roles in sign and energy metabolism, ATP is additionally incorporated into nucleic acids by polymerases within the method of transcription.

Moreover ATP is that the neurochemical believed to signal the sense of taste.

One important reaction (biochemical reaction) is the hydrolysis of ATP into AMP in cells: ATP $\rightarrow$ AMP + PPi.

By this biochemical reaction (ATP hydrolysis) one ATP molecule becomes one AMP molecule and results in addition one pyrophosphate (PPi), which is an anion P2O74- noted with PPi.

The pyrophosphate (diphosphate or dipolyphosphate) anion having structure "P2O74-" is an acid anhydride of phosphate (Fig. 2).

The pyrophosphate is unstable in aqueous solution and hydrolyzes into inorganic phosphate (Hydrogen phosphate, Fig. 3) HPO42- (notted with $\mathrm{Pi}$ ) by reaction: $\mathrm{PPi}+\mathrm{H} 2 \mathrm{O} \rightarrow 2 \mathrm{Pi}$

One phosphate ion (Fig. 4) is a polyatomic ion having the formula PO43- and a mass molar of 94.97 $\mathrm{g} / \mathrm{mol}$. It is builded from one central atom of phosphorus which is surrounded by four atoms of oxygen (in a tetrahedral arrangement). A phosphate ion carries a charge negative-three and is the conjugate base of the hydrogen phosphate ion, HPO42-, who is the base conjugate of H2PO4-, the dihydrogen phosphate ion, which in turn is the conjugate base of $\mathrm{H} 3 \mathrm{PO} 4$, phosphoric acid.

The expert Corey Zah talks about this phosphate ion as the "life's first energy source" (Petrescu and Petrescu, 2015) and we think that he has right.

It has long been known that phosphorus element means energy.

\section{About Mitochondria}

Mitochondria are little organelles present in many eukaryotic cells. Their size generally ranges from 0.5 to 1 micron in diameter. They have shapes and widely varying structures. They are invisible when not dyed specifically to be observed. Mitochondria are named the battery of the life cells, to the extent they contribute to the bulk of the cellular ATP production through the $\beta$ oxidation, the citric acid cycle and oxidative phosphorylation, ATP being the ubiquitous energy molecule used in a very large number of chemical reactions of metabolism, including anabolism.

In addition to their role in cellular energy metabolism, mitochondria are also involved in cell signaling, cell differentiation and cell death, as well as in cell cycle control and cell growth. They have also been associated with several human diseases, such as various heart diseases and mitochondrial diseases. 


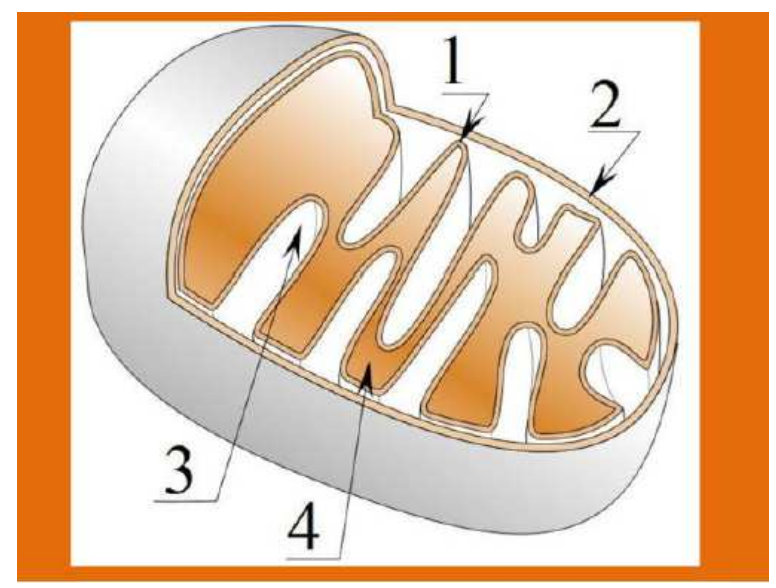

Fig. 5. Mitochondrial structure; Representation of mitochondrial compartments: 1: Internal membrane; 2: External membrane; 3: Intermembrane space; 4: Mitochondrial matrix

Several properties make individual mitochondria organelles. Their number per cell varies widely by species, tissue and cell type. Thus, Red Blood Cells (RBCs) are totally devoid of mitochondria, while the liver cells can contain more than 2000. This organelle is composed of several specialized compartments in several physiological functions: The outer mitochondrial membrane, the space mitochondrial intermembrane, the inner mitochondrial membrane and the mitochondrial matrix. The mitochondrial proteins are dependent species and tissue considered. In humans, cardiac mitochondria contain at least 615 different types of proteins, while it has identified 940 in the rat; the mitochondrial proteome is likely regulated dynamically.

Finally, mitochondria have their own genome, said mitochondrial genome, whose DNA has many analogies with the genome of bacteria.

Mitochondrial structure can be seen on the Fig. 5.

\section{Materials and Methods}

\section{Mitochondrial Genome}

According to symbiogenesis, mitochondria possess a unique monophyletic origin. A primitive prokaryotic cell would have integrated a endosymbiont at about 1.5 to 2 billion years, when the primitive atmosphere was rich in oxygen. Phylogenetic studies indicate that this can be associated with alphaprotéobactéries endosymbiont, the closest relative of mitochondria currently being experienced Rickettsia prowazekii, an obligate intracellular parasite (that is to say, a bacterium that can survive, grow and reproduce that within the cells of its host, using the resources of the latter). During evolution, the majority of the original endosymbiont genes have been lost or transferred to the nucleus of the host eukaryotic cell. Indeed, many mitochondrial pseudogenes present in the genome attest to a transfer process throughout evolution. The genetic material (mtDNA) of the mitochondrion (which is the only part of the animal cell to have its own DNA, in addition to the ring) is frequently used in phylogenetic research. The mitochondrial genome (mtDNA) human is circular, has no introns and consists of 16569 base pairs, including 13 cistrons encoding mRNA, 22 genes encoding transfer RNAs and two genes encoding ribosomal RNA.

The mitochondrial genome can be very different from one species to another, it is extremely dynamic and is often heteroplasmic, that is to say that different forms coexist within the same cell. It can be found in circular or linear, double or single strand. These different forms are, inter alia, the products of the replication of the mitochondrial genome by a rolling circle mechanism, but also a replication-dependent recombination mechanism, similar to the replication of the phage T4. Mitochondrial genomes are usually represented in circular form, a master cycle which is conform with the molecule which may make a best description of genome. Mitochondrial or mitoribosomes ribosomes are different from the cell ribosomes: They are smaller (instead of $80 \mathrm{~S} 70 \mathrm{~S}$ ).

The employee genetic code for protein synthesis can differs from that utilised in cytosolic synthesis. In vertebrates 4 codons 64 have different meanings, the UGA codon that is transcribed in the cytosol stop codon but the UGA template is transcribed in tryptophan (Trp/W), AGG and AGA encoding a STOP codon instead an arginine $(\mathrm{Arg} / \mathrm{R})$ and code AUA Methionine $(\mathrm{Met} / \mathrm{M})$ instead of isoleucine (Ile/I).

Mitochondrial DNA can also replicate.

In animals during sexual reproduction, mitochondria of the sperm into the oocyte could pass, but the number of mitochondria and transferred remains very low compared to those already present in the oocyte. In other words, virtually all of the mitochondria of the egg cell is from the female gamete. The study of the human mitochondrial DNA makes it possible to trace the genealogical relationships between individuals only by mother road.

Some studies have been able to describe an ancestral mitochondrial genome from which descend all mitochondrial genomes of humanity. The individual suspected female who wore this genome was called mitochondrial Eve. This biblical term, however, remains misleading, it is indeed highly unlikely that humanity has a single female ancestor and recent studies proving the transfer of mitochondria from the sperm during fertilization, are challenging this theory.

\section{Mitochondrial Proteome}

Mitochondrial proteome is the totality of the proteins present in the mitochondria of a eukaryotic cell at a 
given time. The proteome is a dynamic set defined in time (time being: Development stage, morning or evening) and space (sample considered: Cell, tissue, organism). To describe the set of proteins that may be present in mitochondria at any time during the life of the body, use the term total proteome. Mitochondrial proteome is composed of proteins produced in the mitochondria and encoded in the mitochondrial genome and of proteins produced in the cytoplasm and encoded in the nuclear genome. Most enzyme complexes (e.g., ATP synthase) are formed by the juxtaposition of polypeptides synthesized within the mitochondrion and in the cytosol (cell's internal fluid). While the mitochondria are the descendants of bacteria, their proteome proteins are not all bacterial, yeast. Thus in the 50 to $60 \%$ of mitochondrial proteins have homologues in prokaryotes, while 40 to $50 \%$ is not. It is interesting to note that thanks to associations of kinesin and dynein protein in the microtubules that mitochondria are capable of movement.

\section{Mitochondrial Proteins Encoded by the Mitochondrial Genome}

From 1 to $10 \%$ of mitochondrial proteins are synthesized directly in the matrix by mitoribosomes, from mitochondrial DNA. Mitochondrial DNA (mtDNA) sequences are widely used for inferring the phylogenetic relationships among species.

The dependence between neighboring nucleotides in a codon make difficile the modeling of nucleotide substitutions in protein-encoding genes.

It seems to be preferable to model the substitution of amino acid than the substitution of nucleotide.

Generally is present a matrix of transition probability of the model reversible Markov of amino acid substitution for mtDNA-encoded proteins.

\section{Mitochondrial Proteins Encoded by the Nuclear Genome}

Mitochondrial protein having a prokaryotic counterpart probably result from the transfer of genes to the nuclear endosymbiont while non-homologous proteins in prokaryotic proteins result from a phenomenon of "enrichment" of the mitochondrial proteome by new proteins and thus new functions. Mitochondrial proteins encoded by the nuclear genome (nuclear or mitochondrial proteins) are imported into the mitochondrial matrix through various possible mechanisms:

- Complex import (3 on the inner membrane, 2 on the outer membrane)

- A signal peptide (about 15 to 30 amino acids) Nterminal position of the protein which permits their recognition and imported into the mitochondrion

\section{- Thanks to energy intake}

\section{Operation}

Mitochondrial is the battery of the cell because that is where unfold the final stages of the respiratory cycle that converts the energy of organic molecules from digestion (glucose) into energy directly usable by the cell (ATP). In case of absence of oxygen the cell uses fermentation in the cytoplasm to produce the energy needed for its operation, but it is a much less efficient system, which degrades the substrate incomplete. Increasing the $\mathrm{H}+$ ion concentration in muscle cells is one of the reasons of fatigue after strenuous activity. Indeed, these $\mathrm{H}+$ ions change the intracellular $\mathrm{pH}$ and enzymatic change of fact operating conditions of the cell can no longer work correctly It is in the mitochondria that are held the last two phases of cellular respiration: The Krebs cycle (in the matrix) and the electron transport chain (at the inner membrane). Indeed, the production of ATP comprises three main steps:

- Glycolysis is the first step. It takes place in the cell cytoplasm

- The second step is the production of acetyl-CoA in the mitochondrion

- The third and final step is the oxidative phosphorylation

During these three stages, via the Krebs cycle (thus aerobic condition), allows the mitochondria, from a glucose molecule, the theoretical production of 36 or 38 molecules of ATP (this depends on the Shuttle used to transport the NAD glycolysis)-in practice, the yield is slightly lower, next thirty molecules of nucleotide per molecule of aldohexose change, some studies giving the value of 29.85 ATP/glucose. Mitochondria participate in apoptosis (cell death) with cytochrome C. In addition, they also have a function of concentration and storage of calcium ions, sodium and potassium where they are stored in the form of opaque granules. There are also gold, iron and osmium.

\section{Mitochondrial Poisons}

Targets poisons Poisons

Complex I; Rotenone; barbiturates; mercury derivatives

Complex II Malonate (malonic acid)

Complex III Antimycin

Complex IV; Azote monoxide; Cyanide; Carbon monoxide

Complex V (F0/F1ATPase) oligomycin; Aurovertine

Exchanger ATP/ADP atractyloside; bongkrekic acid

Permeability of the inner membrane Dinitrophenol; valinomycin 
Some fish have the function not to prevent the different complexes to function, that is to say, the electron transfer in the respiratory chain are made but these proteins, uncoupling or PCU will bypass the complex V (ATP synthase) creating a channel through the inner membrane. This pore allows protons to pass from the inter-membrane space to the matrix in the way of the own gradient, that allow obtains an exothermic reaction but no ATP production. We can quote the example of dinitrophenol.

\section{Oxidative Phosphorylation}

Oxidative phosphorylation is oxygen consumption (mitochondrial respiration) associated with phosphorylation of Adenosine Diphosphate (ADP) Adenosine Triphosphate (ATP). The oxygen consumed acts as terminal electron acceptor and proton. Attaching a phosphate to ADP absorbs a certain amount of energy. The ATP are chemical energy accumulators, outgoing mitochondria and participating in endothermic reactions as they provide the energy needed by losing again becoming a phosphate and ADP. The image centenary molecules of fat and sugars "burning" in the cells to provide energy needed for major biological phenomena, movement, growth, proliferation, defense, is explained: These molecules do not burn by direct combination with $\mathrm{O} 2$, resulting in an immediate release and total energy raising the temperature to values incompatible with life. A molecule by direct reaction reduced oxygen with 4 hydrogen atoms releases $483.2 \mathrm{~kJ}$ (Equation 1):

$$
\mathrm{O}_{2}+4 e^{-}+4 \mathrm{H}^{+} \rightarrow 2 \mathrm{H}_{2} \mathrm{O}+115.6 \mathrm{kCal}
$$

The energy of this reaction in vitro (combustion of hydrogen in air jet) is very high, causing a very high temperature, incompatible with life: It emerges 115.6 $\mathrm{kcal}$ or $483.2 \mathrm{~kJ}$ or $3.016 \mathrm{E} 18 \mathrm{MeV}(1 \mathrm{~kJ}=$ $6.2415096471204 \mathrm{E} 15 \mathrm{MeV})$. The potential energy of the metabolite will be slowly released by a cascade of enzymatic reactions that dissipate the "packets" of less than $100 \mathrm{~kJ}$ will be immediately used for the phosphorylation of ADP molecules. After this chain of reactions, an oxygen molecule will accept 4 electrons from low energy levels and four protons.

The normal pressure of oxygen at the mitochondrion is $0.3 \mathrm{kPa}$ ( 2 Torr). Isolated mitochondria exposed to atmospheric oxygen pressure realizes, as can see, a strong hyperoxia. Three steps can be distinguished in these complex phenomena:

- The formation of "pools" of enzyme co-factors: FADH2 and NADH; the Krebs cycle taking place in the matrix ensures their reduction while providing protons and high energy electrons. These cofactors are essential to the operation of the cascade of reactions by releasing energy levels of the electrons; they are the two entry gates of the electron transport chain

- Transport of electrons to oxygen or ETC

- Continuous absorption of "packets" of energy for the phosphorylation of ADP, by the ATP synthesis

Oxidative phosphorylation (OxyPhos) takes place in the complex V $(\mathrm{CoV})$ or ATP synthase which fragments the energy released during the transport of electrons and accumulates by creating a high-energy bond with a phosphate group ( $P_{i}$ for inorganic phosphate), during the enzymatic reaction represented by Equation 2:

$A D P+P_{i}+e n e r g y \rightarrow A T P$

$P_{i}$ is present as a balanced mixture of phosphates represented in Equation 3:

$\mathrm{H}_{2} \mathrm{PO}^{-}$and $\mathrm{HP}_{4}^{2-}$

These ATP molecules are small energy accumulators which spread into the cytosol and enable many endothermic reactions to occur. Four metallo protein complexes are fixed in the inner membrane: Complex I (CoI), complex II (CoII), the complex III (CoIII) and complex IV (CoIV). The electrons released by the intramatrix Krebs cycle enter through two portals in the respiratory chain enzyme system NADH oxidase FMN attached to the CoI and FAD succinate dehydrogenase fixed on the CoII. The two streams of electrons result in a small molecule attached in the inner membrane, the Coenzyme Q10 (ubiquinone 10). Each of the two complexes (CoI and CoII) gives its electrons to ubiquinone (Q10). From there, e- enter in the complex III and reduce the heme iron of mobile molecules of cytochrome c. These are then oxidized by the CoIV and return seek an electron from the CoIII. Cytochrome c acts as a transport shuttle, operating in the intermembrane space. Finally, the electrons are used by the matrix pole of CoIV to reduce an oxygen molecule into two molecules of water. Complex V or ATP synthase or F0-F1. Complex V is the principal place of synthesis of cellular ATP.

Melatonin and its metabolite N-acetyl-5méthoxykynurénamine (AMK) physiologically would regulate the ETC and oxidative phosphorylation. Melatonin counteract the inhibitory effect of LPS on I and IV complex that occurs by excess production *NO, its effect being the more important on animal and human age. The interpretation given to explain the behavior of this molecule is its antioxidant action. To remember! Coenzyme Q10 (ubiquinone 10) and Melatonin. 


\section{Results}

\section{Mitochondrial Redox Phenomena}

Tissues and cells need maintain a reducing environment to survive. The energy required for life is provided by a continuous flow of electrons between the oxidized metabolites (able to lose electrons) and diatomic oxygen ultimate goal of their movement. These electrons are carriers of energy whose value is given by measuring the potential difference between the carrier molecule of the electron (reduced state) and even after the departure of e (oxidized state), measured in millivolts. Measurement by voltmeter recorder shows a sigmoid curve; the middle potential (mid-potential) passes through the inflexion point of the curve. ETC thus comprises steps characterized by reduced/oxidized couples having midpotentials less negative (or more positive). The growth of the algebraic value of the electric potential is, as we shall see, to an expressible release of energy in electron volts, but also in Joule or kcal. The flow of electrons occurs due to the existence of redox couples cascades in cells whose potential, very negative to the top of the cascade, gradually becomes positive. But chemical agents that oxidations are activated forms of the $\mathrm{O} 2$ (ROS or reactive oxygen species). There is a quarter century there was talk of the cellular redox potential and redox state. Today, the term of redox state is given to one special redox couple who has its median potential (mid potential), also called halfcell potential (half-potential), measured under standard conditions (molarity: 1; pressure: 1 atm; temperature: $25^{\circ} \mathrm{C} ; \mathrm{pH} 0$ ) and its reductive capacity. The cellular redox environment is an essential factor for various metabolic cell functions. It is provided mainly by the balance between reduced forms of - $\mathrm{SH}$ thiol functions and their oxidized forms -S-S- (disulfide). Main mitochondrial redox couples:

- $\quad \mathrm{GSSG} / 2 \mathrm{GSH}$

- $\mathrm{NAD}^{+} / \mathrm{NADH}$

- $\mathrm{NADP}^{+} / \mathrm{NADPH}$

- $\mathrm{CoQ} / \mathrm{CoQ} *$

The energy released during the reduction of an oxygen atom. Consider the "half-reaction" of oxygen reduction in the presence of protons:

$$
2 \mathrm{H}^{+}+\frac{1}{2} \mathrm{O}_{2} \rightarrow \mathrm{H}_{2} \mathrm{O}+0.816 \mathrm{~V}
$$

It can be compared to the starting redox reaction at the CoI ETC: $\mathrm{NAD}^{+} / \mathrm{NADH}$, for which $\mathrm{E} 0=-0.32 \mathrm{~V}$. The energy released by the half-reaction (4) is considerable and lead the environment in which it happens to combustion (direct reaction of oxygen with a combustible material) If the energy supplied by the electrons was not previously weakened. In nature, it is in small quantities what this dissipated energy that could be explosive. The electrons become less and less energy. ROS (Reactive Oxygen Species) have a very specific physiological role in the production of signals occurring in the cell redox potential. A brief overview on thiolated molecules and selenium (They achieve regulatory systems of the redox potential). In the following series of reactions (5), "ROS" (Reactive Oxygen Species) symbolizes various activated derivatives of molecular oxygen:

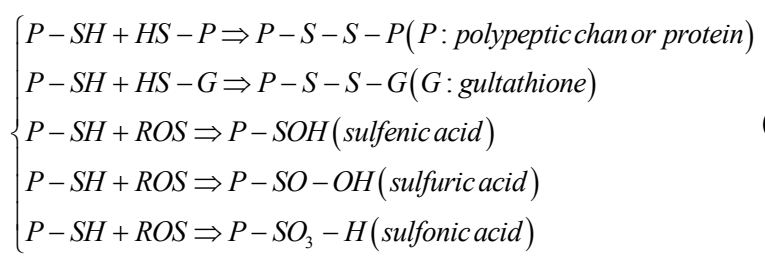

These cysteinyl residues are controlled by the thioredoxin and glutaredoxins systems.

\section{Glutathione}

Glutathione is the major part of the low molecular weight thiols involved in redox homeostasis in mammalian cells and plays a central role in cellular defense. Mitochondrial glutathione alone is an important part of the reserve cell GSH: 10 to $15 \%$ of the total glutathione in the liver and renal proximal tubules. Mitochondria import GSH cytosol and store almost 15\% of total cellular GSH content. Regeneration of GSH shape is the center of the regulating of the redox potential of a mitochondrion. There is a symbiosis between this process and the NADPH regeneration cycles and NADH and the role of transhydrogenase.

\section{The Thioredoxin $(\operatorname{Tr} x)$}

Trx Thioredoxins are enzymes intrinsically antioxidant activity like all protein thiol group (-SH). They also play an important role in regulating the immune system. Once oxidized thioredoxin is reduced by thioredoxin reductase (TrxR). It was in 1964 that was first isolated Trx. These are small proteins of $12 \mathrm{kDa}$ having an active site dithiol, which are part of an antioxidant system which the first known members are glutathione (G-SH) and glutathione peroxidase. Both cysteinyl residues oxidized (inactive) may be reduced by a flavoenzyme, thioredoxin reductase (TrxR) in the presence of NADPH (Zhou et al., 2007). Thioredoxins also protect the cell against the electrophiles. Mammalian cells contain two types of Trx; TRX1 is present in the cytosol, while TRX2 is localized in the mitochondria. The Trxs are electron donors for carrying enzymes two thiol groups capable of forming a disulfide bond, such as peroxiredoxins. Mitochondrial 
thioredoxin-2 has been cloned from the mitochondrial DNA of the heart muscle, liver, stem cells and placenta.

\section{The Thioredoxin Reductase (TrxR)}

The thioredoxin reductase (TrxR) are enzymes with a selenocysteine group in their active site. The TrxR also involved in the degradation of lipid peroxides and hydrogen peroxide and in the regeneration of the ascorbyl radical in ascorbic acid. Both mammalian thioredoxin reductase, TrxR-1 and TrxR-2 are enzymes FAD, homodimeric (54-58 $\mathrm{kDa})$, to selenocysteine and the two components each have the same catalytic site. They catalyze the reduction by NADPH oxidized thioredoxin disulfide. They are inhibited by curcumin. In cooperation with thioredoxin, they contribute to regulation of mitochondrial and cytosolic redox potential.

\section{Disorders of the Redox Balance and Activation of O2}

For various reasons, the redox equilibrium can be brought into a more positive area, so oxidizing that normally and it is called oxidative stress. This is a pathological situation and is produced by ROS when the concentration exceeds the limits within which they play a physiological role. In other situations, the redox balance may become more negative, more reductive: This is called stress reducer and may occur in that moment when the redox environment becomes too negative and is rooted both in the cytosol and in the mitochondria. In both cases, direct activation of the oxygen or the appearance of activating radical reactions of oxygen will occur. Disorders of redox balance damages the oxidative cellular macromolecules. Today was shown that these disorders can be made and by oxidative stress and by stressing the animal or human. Such a house that collapses if one crush bricks components and animal or human body can be sick by disruption of cell components.

\section{Discussion}

Mitochondrial diseases or mitochondriopathies linking together a patchwork of diseases associated with mitochondrial respiratory chain disorders. This disorder is secondary to a mutation of nuclear or mitochondrial DNA.

Some more general diseases involve mutations of mitochondrial DNA, although the exact role of the latter is not known: It is the case in particular Parkinson's disease and Alzheimer's disease. Other diseases probably have a mitochondrial participation whose cause remains hypothetical. This is the case so certain cardiomyopathies, of Huntington's disease or obesity with resistance insuline.

The involvement of mitochondria in energy production chain accounts for a preferential involvement of organs highly dependent on the latter: Neurons, muscles.

More than 1,000 genes involved in mitochondrial function. At the date of 2012, the causal mutations of mitochondrial disease have been described in humans in more than 200 nuclear genes and mitochondrial genes 13 .

Some mitochondrial diseases reach only one organ (like the eye in Leber hereditary optic neuropathy), but most involve many organs and muscles with neurological signs often in the foreground.

The manifestations of these diseases occur at any age. In general, mutations in nuclear DNA occur earlier than mutations of mitochondrial DNA.

However, the great variability of clinical manifestations does not always allow for an accurate diagnosis. The most common signs of mitochondropathie are ptosis, external ophthalmoplegia, myopathy or excessive muscle fatigue, cardiomyopathy, decreased vision and hearing, optic atrophy, retinitis pigmentosa or diabetes.

The most common neurological signs are encephalopathy, epilepsy, dementia, ataxia and spastic disorders.

Physiologic, optimal concentrations may exist, producing the normal effect, favorable to the smooth running of cellular life.

The refinement of molecular biology techniques and the deepening of mitochondrial biochemistry helped make a distinction quite revolutionary for the pioneers of oxygen metabolism. ROS play an indispensable role in this newly discovered function: The signal transmission. Let us briefly recall that a cell is part of a fabric and that this set may according to its specialization, play a role, to multiply, to contract or relax etc. In times of growth, cell groups should disappear by apoptosis.

Extracellular messengers (messengers or humoral first messengers) trigger a signal by pressing a transmembrane receptor, which in structure changing will initiate a signal that will propagate in the cell to the effector sites, with intracellular second messengers. Through these mechanisms, coordination is established in the constitutive behavior of tissue cells. Mitochondria are often involved in these processes, including apoptosis, ROS and use as normal steps.

\section{Conclusion}

One phosphate ion is a polyatomic ion having the formula PO43- and a mass molar of $94.97 \mathrm{~g} / \mathrm{mol}$. It is builded from one central atom of phosphorus which is surrounded by four atoms of oxygen (in a tetrahedral arrangement). A phosphate ion carries a charge negativethree and is the conjugate base of the hydrogen phosphate ion, HPO42-, who is the base conjugate of H2PO4-, the dihydrogen phosphate ion, which in turn is the conjugate base of H3PO4, phosphoric acid. 
Mitochondrion can only come from the growth and division of another existing mitochondrion. Normally, before cell division, mitochondrion doubles its mass and then splits into two. This division begins with the appearance of a cleavage furrow on the inner membrane. It takes place during interphase and requires the intervention of the DRP1 protein. Mitochondrial DNA replication is not limited to the $\mathrm{S}$ phase of the cell cycle. The number of mitochondria per cell is regulated by cellular activity. For example, a muscle cell break contains 5 to 10 times less than a Mitochondria permanently activated muscle cell.

The fact that the mitochondrion has its own DNA, such as chloroplasts, indicates an exogenous origin: It is now accepted that mitochondria come from the endosymbiosis of an $\alpha$-proteobacteria there are about 2 billion years. The symbiogenesis the origin of mitochondria was developed and argued by Lynn Margulis in 1966 and it was supported by the discovery of the specific mitochondrial DNA in 1980. It appears that during evolution the original DNA of the bacterium has undergone various changes, lost a large number of genes, sometimes transferred to the DNA of the host cell. Parallel to this report the synthesis of certain proteins to the host, the host has developed a battery of translocases, enzymes enabling the transfer of these proteins to the mitochondrial matrix.

Years ago the medical specialists studying organ disease have considered that is caused by the impaired organ or related to other organs. For example, it was considered that all nerve diseases have starting from stress due to disorder of the hypothalamic-pituitaryadrenal (especially adrenal glands).

Today the experience of psychological stress triggers neuroendocrine, inflammatory, metabolic and transcriptional perturbations which finally conduct to diseases (However, the subcellular determinants of this integrated, multisystemic stress response have not been defined) and central to stress adaptation is cellular energetics, involving mitochondrial energy production and oxidative stress.

All future research on mitochondrial, may lead tomorrow to elucidation of great issues of life (including the generation of illness, aging and death bodies). The main idea of this paper is that mitochondria are independent cells, flexible, essential for complex life. Mitochondrial ROS are detoxified by a specialized intramitochondrial antioxidant system maintained by the redox enzyme (nicotinamide nucleotide transhydrogenase NNT).

Another important function of a mitochondrion is to kill eukaryotic cells when it is their time to die. If a eukaryotic cell can multiply by 40 times when multiplied by 39 times the mitochondria present in it already begin to prepare the eukaryotic cell for death. They begin to leave the eukaryotic cell to perdition, not to die with her and to no longer supply her with power. If one eukaryotic cell is sick the inside mitochondrial cells are trying to heal it first, but if this is not possible then the eukaryotic cell is marked and all mitochondria inside begin the process to kill her even the eukaryotic cell still has many multiplication cycles possible. If eukaryotic cell (dying, abandoned by mitochondria) do not dies quickly, turns into a diseased cell cancer (harmful to the body). The same undesirable phenomenon can happen when they get sick mitochondrial cells itself.

\section{Acknowledgement}

This text was acknowledged and appreciated by Assoc. Pro. Taher M. Abu-Lebdeh, North Carolina A and T State Univesity, United States, Muftah H. ElNaas PhD MCIC FICCE QAFCO Chair Professor in Chemical Process Engineering Gas Processing Center College of Engineering Qatar University, Professor Guanying Chen Harbin Institute of Technology and SUNY Buffalo China and (Ms.) Shweta Agarwala Senior Research Scientist at Singapore Center for 3D Printing Nanyang Technological University Singapore, whom we thanks and in this way.

\section{Author's Contributions}

All the authors contributed equally to prepare, develop and carry out this manuscript.

\section{Ethics}

This article is original and contains unpublished material. The corresponding author confirms that all of the other authors have read and approved the manuscript and no ethical issues involved.

\section{References}

Andrews, R.C. and B.R. Walker, 1999. Glucocorticoids and insulin resistance: Old hormones, new targets. Clin. Sci., 96: 513-523. DOI: 10.1042/cs0960513

Arkblad, E.L., C. Betsholtz and J. Rydström, 1996. The cDNA sequence of proton-pumping nicotinamide nucleotide transhydrogenase from man and mouse. Biochim. Biophys. Acta, 1273: 203-205. DOI: $10.1016 / 0005-2728(95) 00159-X$

Bandura, A., C.B. Taylor, S.L. Williams, I.N. Mefford and J.D. Barchas, 1985. Catecholamine secretion as a function of perceived coping self-efficacy. J. Consult Clin. Psychol., 53: 406-414. DOI: 10.1037/0022-006X.53.3.406

Booth, F.W., M.J. Laye, S.J. Lees, R.S. Rector and J.P. Thyfault, 2008. Reduced physical activity and risk of chronic disease: The biology behind the consequences. Eur. J. Applied Physiol., 102: 381-390. DOI: $10.1007 / \mathrm{s} 00421-007-0606-5$ 
Bornstein, S.R., W.C. Engeland, M. Ehrhart-Bornstein and J.P. Herman, 2008. Dissociation of ACTH and glucocorticoids. Trends Endocrinol. Metab., 19: 175-180. DOI: 10.1016/j.tem.2008.01.009

Bullitt, E., 1990. Expression of $C$-fos-like protein as a marker for neuronal activity following noxious stimulation in the rat. J. Comp. Neurol., 296: 517-530. DOI: $10.1002 /$ cne. 902960402

Buzea, E., F.L. Petrescu, L. Nănuţ, C. Nan and M. Neacşa, 2015. Mechatronic system to determine the concentration of carotenoids. Analele Universitatii Din Craiova Biologie Horticultura Tehnologia Prelucrarii Produselor Agricole Ingineria Mediului, 20: 371-376.

Cohen, S., D. Janicki-Deverts and G.E. Miller, 2007. Psychological stress and disease. JAMA, 298: 1685-1687. DOI: 10.1001/jama.298.14.1685

DeSantis, A.S., E.K. Adam, L.C. Hawkley, B.M. Kudielka and J.T. Cacioppo, 2015. Racial and ethnic differences in diurnal cortisol rhythms: Are they consistent over time? Psychosom. Med., 77: 6-15. DOI: 10.1097/PSY.0000000000000131

Dimsdale, J.E. and J. Moss, 1980. Plasma catecholamines in stress and exercise. JAMA, 243: 340-342. DOI: 10.1001/jama.1980.03300300018017

Dimsdale, J.E. and J.A. Herd, 1982. Variability of plasma lipids in response to emotional arousal. Psychosom. Med., 44: 413-430. DOI: $10.1097 / 00006842-198211000-00004$

Gimsa, U., E. Kanitz, W. Otten and S.M. Ibrahim, 2009. Behavior and stress reactivity in mouse strains with mitochondrial DNA variations. Ann. N Y Acad. Sci., 1153: 131-138. DOI: 10.1111/j.1749-6632.2008.03960.x

Gómez-Durán, A., D. Pacheu-Grau, E. López-Gallardo, C. Díez-Sánchez and J. Montoya et al., 2010. Unmasking the causes of multifactorial disorders: OXPHOS differences between mitochondrial haplogroups. Hum. Mol. Genet., 19: 3343-3353. DOI: $10.1093 / \mathrm{hmg} / \mathrm{ddq} 246$

Gonzalez-Bono, E., N. Rohleder, D.H. Hellhammer, A. Salvador and C. Kirschbaum, 2002. Glucose but not protein or fat load amplifies the cortisol response to psychosocial stress. Horm. Behav., 41: 328-333. DOI: 10.1006/hbeh.2002.1766

Gorman, G.S., A.M. Schaefer, Y. Ng, N. Gomez and E.L. Blakely et al., 2015. Prevalence of nuclear and mitochondrial DNA mutations related to adult mitochondrial disease. Ann. Neurol., 77: 753-759. DOI: 10.1002/ana.24362

Gray, J.D., T.G. Rubin, R.G. Hunter and B.S. McEwen, 2014. Hippocampal gene expression changes underlying stress sensitization and recovery. Mol. Psychiatry, 19: 1171-1178.

DOI: $10.1038 / \mathrm{mp} .2013 .175$
Gut, P. and E. Verdin, 2013. The nexus of chromatin regulation and intermediary metabolism. Nature, 502: 489-498. DOI: 10.1038/nature 12752

Hunter, R.G., G. Murakami, S. Dewell, M. Seligsohn and M.E.R. Baker et al., 2012. Acute stress and hippocampal histone H3 lysine 9 trimethylation, a retrotransposon silencing response. Proc. Nat. Acad. Sci. USA., 109: 17657-17662.

DOI: 10.1073 /pnas. 1215810109

Jeppesen, T.D., M.C. Orngreen, G. Van Hall and J. Vissing, 2013. Lactate metabolism during exercise in patients with mitochondrial myopathy. Neuromuscul. Disord., 23: 629-636. DOI: 10.1016/j.nmd.2013.05.007

Jones, B.N. and J.P. Gilligan, 1983. o-Phthaldialdehyde precolumn derivatization and reversed-phase highperformance liquid chromatography of polypeptide hydrolysates and physiological fluids. J. Chromatogr. A, 266: 471-482.

DOI: 10.1016/S0021-9673(01)90918-5

Juster, R.P., A. Perna, M.F. Marin, S. Sindi and S.J. Lupien, 2012. Timing is everything: Anticipatory stress dynamics among cortisol and blood pressure reactivity and recovery in healthy adults. Stress, 15 : 569-577. DOI: 10.3109/10253890.2012.661494

Kado, S., T. Nagase and N. Nagata, 1999. Circulating levels of interleukin-6, its soluble receptor and interleukin-6/interleukin-6 receptor complexes in patients with type 2 diabetes mellitus. Acta Diabetol., 36: 67-72. DOI: 10.1007/s005920050147

Koopman, W.J., P.H. Willems and J.A. Smeitink, 2012. Monogenic mitochondrial disorders. $\mathrm{N}$ Engl. J. Med., 366: 1132-1141.

DOI: $10.1056 /$ NEJMra1012478

Lambert, A.J. and M.D. Brand, 2009. Reactive oxygen species production by mitochondria. Meth. Mol. Biol., 554: 165-181. DOI: $10.1007 / 978-1-59745-521-3 \_11$

Livak, K.J. and T.D. Schmittgen, 2001. Analysis of relative gene expression data using real-time quantitative PCR and the 2(-Delta Delta C(T)) method. Methods, 25: 402-408. DOI: 10.1006/meth.2001.1262

Lundberg, U., 2005. Stress hormones in health and illness: The roles of work and gender. Psychoneuroendocrinology, 30: 1017-1021. DOI: $10.1016 /$ j.psyneuen.2005.03.014

Manoli, I., S. Alesci, M.R. Blackman, Y.A. Su and O.M. Rennert et al., 2007. Mitochondria as key components of the stress response. Trends Endocrinol. Metab., 18: 190-198. DOI: $10.1016 /$ j.tem.2007.04.004

McEwen, B.S., 1998a. Protective and damaging effects of stress mediators. N Engl. J. Med., 338: 171-179. DOI: 10.1056/NEJM199801153380307

McEwen, B.S., 1998b. Stress, adaptation and disease: Allostasis and allostatic load. Ann. N Y Acad. Sci., 840: 33-44. DOI: 10.1111/j.1749-6632.1998.tb09546.x 
McEwen, B.S., 2007. Physiology and neurobiology of stress and adaptation: Central role of the brain. Physiol. Rev., 87: 873-904.

DOI: 10.1152 /physrev.00041.2006

McFarland, R., R.W. Taylor and D.M. Turnbull, 2010. A neurological perspective on mitochondrial disease. Lancet Neurol., 9: 829-840. DOI: $10.1016 / \mathrm{S} 1474-4422(10) 70116-2$

Meimaridou, E., C.R. Hughes, J. Kowalczyk, L.F. Chan and A.J.L. Clark et al., 2013. ACTH resistance: Genes and mechanisms. Endocr. Dev., 24: 57-66. DOI: $10.1159 / 000342504$

Morava, E. and T. Kozicz, 2013. Mitochondria and the economy of stress (mal)adaptation. Neurosci. Biobehav. Rev., 37: 668-680.

DOI: 10.1016/j.neubiorev.2013.02.005

Nasca, C., B. Bigio, D. Zelli, F. Nicoletti and B.S. McEwen, 2015. Mind the gap: Glucocorticoids modulate hippocampal glutamate tone underlying individual differences in stress susceptibility. Mol. Psychiatry, 20: 755-76. DOI: 10.1038/mp.2014.96

Olsen, R.H., L.A. Johnson, D.G. Zuloaga, C.L. Limoli and J. Raber, 2013. Enhanced hippocampusdependent memory and reduced anxiety in mice over-expressing human catalase in mitochondria. J. Neurochem., 125: 303-313. DOI: 10.1111/jnc.12187

Pelletier, M., T.S. Lepow, L.K. Billingham, M.P. Murphy and R.M. Siegel, 2012. New tricks from an old dog: Mitochondrial redox signaling in cellular inflammation. Semin. Immunol., 24: 384-392. DOI: $10.1016 /$ j.smim.2013.01.002

Petrescu, F. and R. Petrescu, 2015. Alternative energy magazine.

Petrescu, FL., E. Buzea, L. Nănuţ, M. Neacşa and C. Nan, 2015. The role of antioxidants in slowing aging of skin in a human. Analele Universitatii Din Craiova Biologie Horticultura Tehnologia Prelucrarii Produselor Agricole Ingineria Mediului, 20: 567-574.

Picard, M. and D.M. Turnbull, 2013. Linking the metabolic state and mitochondrial DNA in chronic disease, health and aging. Diabetes, 62: 672-678. DOI: $10.2337 / \mathrm{db} 12-1203$

Picard, M. and B.S. McEwen, 2014. Mitochondria impact brain function and cognition. Proc. Nat. Acad. Sci. USA., 111: 7-8. DOI: 10.1073/pnas.1321881111

Picard, M., R.P. Juster and B.S. McEwen, 2014a. Mitochondrial allostatic load puts the 'gluc' back in glucocorticoids. Nat. Rev. Endocrinol., 10: 303-310. DOI: $10.1038 /$ nrendo.2014.22

Picard, M., J. Zhang, S. Hancock, O. Derbeneva and R. Golhar et al., 2014b. Progressive increase in mtDNA 3243A $>\mathrm{G}$ heteroplasmy causes abrupt transcriptional reprogramming. Proc. Nat. Acad. Sci. USA., 111: E4033-E4042.

DOI: $10.1073 /$ pnas. 1414028111
Picard, M., M.J. McManus, J.D. Gray, C. Nasca and C. Moffat et al., 2015. Mitochondrial functions modulate neuroendocrine, metabolic, inflammatory and transcriptional responses to acute psychological stress. PNAS, 112: E6614-E6623. DOI: $10.1073 /$ pnas. 1515733112

Rangaraju, V., N. Calloway and T.A. Ryan, 2014. Activity-driven local ATP synthesis is required for synaptic function. Cell, 156: 825-835.

DOI: $10.1016 /$ j.cell.2013.12.042

Rohleder, N., 2014. Stimulation of systemic low-grade inflammation by psychosocial stress. Psychosom. Med., 76: 181-189.

DOI: 10.1097/PSY.0000000000000049

Saudubray, J.M. and D. Rabier, 2007. Biomarkers identified in inborn errors for lysine, arginine and ornithine. J. Nutr., 137: 1669S-1672S. PMID: 17513445

Schroeder, E.A., N. Raimundo and G.S. Shadel, 2013. Epigenetic silencing mediates mitochondria stressinduced longevity. Cell Metab., 17: 954-964. DOI: $10.1016 /$ j.cmet.2013.04.003

Sindi, S., A.J. Fiocco, R.P. Juster, J. Pruessner and S.J. Lupien, 2013. When we test, do we stress? Impact of the testing environment on cortisol secretion and memory performance in older adults. Psychoneuroendocrinology, 38: 1388-1396. DOI: 10.1016/j.psyneuen.2012.12.004

Singh, A., J.S. Petrides, P.W. Gold, G.P. Chrousos and P.A. Deuster, 1999. Differential hypothalamicpituitary-adrenal axis reactivity to psychological and physical stress. J. Clin. Endocrinol. Metab., 84: 1944-1948. PMID: 10372691

Sun, T., H. Qiao, P.Y. Pan, Y. Chen and Z.H. Sheng, 2013. Motile axonal mitochondria contribute to the variability of presynaptic strength. Cell Rep., 4: 413-419. DOI: 10.1016/j.celrep.2013.06.040

Szendroedi, J., E. Phielix and M. Roden, 2012. The role of mitochondria in insulin resistance and type 2 diabetes mellitus. Nat. Rev. Endocrinol., 8: 92-103. DOI: $10.1038 /$ nrendo.2011.138

Ulrich-Lai, Y.M. and J.P. Herman, 2009. Neural regulation of endocrine and autonomic stress responses. Nat. Rev. Neurosci., 10: 397-409. DOI: 10.1038/nrn2647

Vos, M., E. Lauwers and P. Verstreken, 2010. Synaptic mitochondria in synaptic transmission and organization of vesicle pools in health and disease. Front Synaptic Neurosci., 2: 1-10. DOI: 10.3389/fnsyn.2010.00139

Wallace, D.C., 2013. A mitochondrial bioenergetic etiology of disease. J. Clin. Invest., 123: 1405-1412. DOI: 10.1172/JCI61398

Wallace, D.C., W. Fan and V. Procaccio, 2010. Mitochondrial energetics and therapeutics. Ann. Rev. Pathol., 5: 297-348.

DOI: 10.1146/annurev.pathol.4.110807.092314 
Weiner, H., 1992. Perturbing the Organism: The Biology of Stressful Experience. University of Chicago Press, ISBN-10: 0226890414, pp: 357.

Wellen, K.E., G. Hatzivassiliou, U.M. Sachdeva, T.V. Bui and J.R. Cross et al., 2009. ATP-citrate lyase links cellular metabolism to histone acetylation. Science, 324: 1076-1080. DOI: 10.1126/science.1164097

Xia, J., R. Mandal, I.V. Sinelnikov, D. Broadhurst and D.S. Wishart, 2012. MetaboAnalyst 2.0--a comprehensive server for metabolomic data analysis. Nucleic Acids Res., 40: W127-133. DOI: 10.1093/nar/gks374
Ye, K., J. Lu, F. Ma, A. Keinan and Z. Gu, 2014. Extensive pathogenicity of mitochondrial heteroplasmy in healthy human individuals. Proc. Nat. Acad. Sci. USA., 111: 10654-10659. DOI: 10.1073/pnas.1403521111

Zhang, Q., M. Raoof, Y. Chen, Y. Sumi and T. Sursal et al., 2010. Circulating mitochondrial DAMPs cause inflammatory responses to injury. Nature, 464: 104-107. DOI: 10.1038 /nature08780

Zhou, J., A.E. Damdimopoulos, G. Spyrou and B. Brune, 2007. Thioredoxin 1 and thioredoxin 2 have opposed regulatory functions on hypoxia-inducible factor-1 $\alpha$. J. Biol. Chem., 282: 7482-7490.

DOI: $10.1074 /$ jbc.M608289200 